\title{
Research on Traffic Sign Recognition Algorithm based on SVM of LBP
}

\author{
Ziyi Zhou \\ University of Electronic Science and Technology of China. \\ 598326927@qq.com
}

\begin{abstract}
For the problem of road traffic sign, this paper proposed the traffic sign recognition algorithm based on SVM of LBP. Firstly, colour space conversion, binarization processing, expansion, demonizing and filling are carried out for the collected signs to obtain the target area in the image. Then, LBP operator is used to obtain the texture features of the target area, and then the features are input into SVM for training. Finally, the traffic signs are identified with the SVM after training, and compared with the traffic recognition database.
\end{abstract}

Keywords: Texture feature; SVM; LBP; Traffic sign recognition.

\section{Introduction}

With the development of road traffic and the popularization of vehicles, road traffic safety has become an issue of public concern. Therefore, for the protection of driver's life safety and traffic road safety, the computer recognition system with traffic intelligent recognition function has emerged. Its role is to automatically recognise traffic conditions on the road, replace drivers to recognise traffic signs, make drivers more focused on driving, alert drivers to make emergency reaction to potential hazards and keep them in the right lane. Therefore, the research on intelligent recognition of traffic signs can greatly improve the safety and smoothness of road traffic and ensure the safety of drivers.

Traffic sign recognition is generally divided into two steps: detection and classification. The purpose of detection is to find the location and size of existing traffic signs in the image. The task of classification is to assign a class label to each class. A great deal of research on traffic sign recognition has been carried out at home and abroad. In terms of detection, the common method is to convert the image into a new colour space. But in complex environments, such method has limitations and unpredictable lighting conditions. References [15] and [16] used the sliding window-based method to detect traffic signs. However, this method is very time-consuming because a multi-scale pyramid needs to be constructed and it is difficult to determine the size of sliding window and its aspect ratio. References [17] and [18] put forward the method based on regional interest. Compared with the method based on sliding window, such method reduces calculation time and does not need to adjust parameters, but it requires guaranteeing the recall rate of ROI target object. For the classification of traffic signs, the traditional classification method includes feature extraction and classifier training. This kind of method is very complex and difficult to make features. References [19] applied the trainable drama neural network method, which does not require manual design and performs well in the classification of traffic signs by different weight initialization or data pre-processing recommendations.

This paper will discuss the traffic sign recognition algorithm based on SVM of LBP. Firstly, the image is pre-processed, converted to binary image and expanded. Then the binary image is filled with road sign image. Eliminate non-target area, identify the area of interest, and lock the position of road traffic sign. Finally, the results are brought into the LBP-based SVM to recognize the traffic signs. 


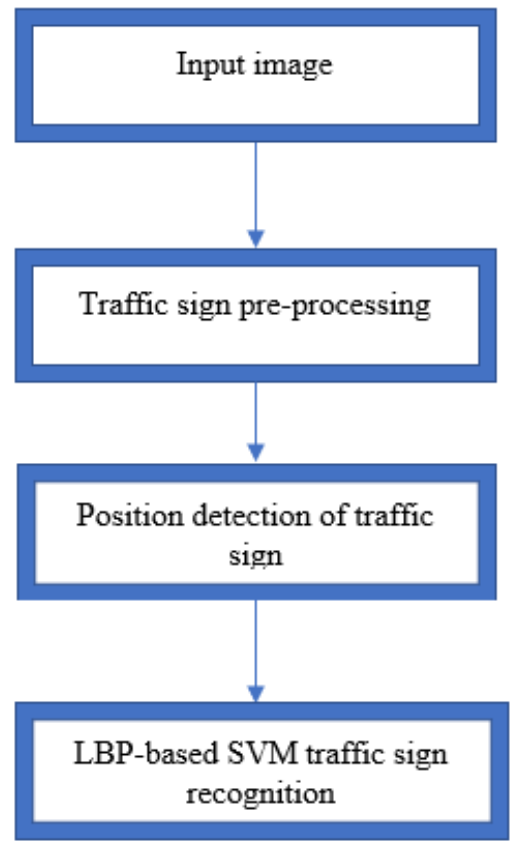

Fig 1. The process

\section{Traffic Sign Detection}

\subsection{HSV Color Space Segmentation}

Commonly used colour space is RGB and HSV space. RGB space is the most commonly used colour space expression of digital images, but it cannot be directly reflected in the recognition of human eyes. The three channels of HSV space: $\mathrm{H}$ stands for colour, S stands for saturation, and V stands for value, which is more consistent with human eyes' subjective understanding of colour. Therefore, the image to be tested is first transformed into HSV space image.

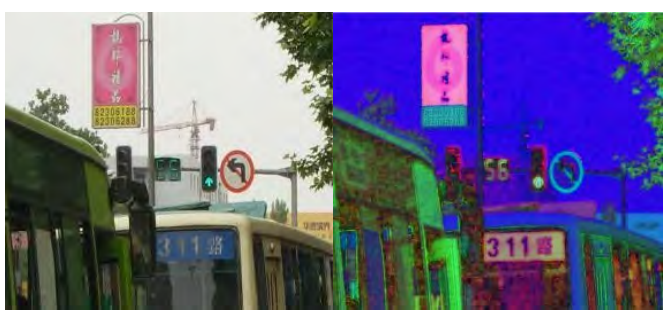

Fig 2. (a)Original image (b) HSV space

\subsection{Colour Binarization of Road Signs}

Any colour is determined by adding the components R, G, and B. But one downside is that they are highly correlated. When the illumination changes, it is not good to purely use RGB colour space to segment the image. Therefore, in order to reduce the interference, the differences between $R, G$ and $\mathrm{B}$ components are used :(r-g), (g-b) and (b-r) are used determine the colour. The segmentation threshold is: red: $(\mathrm{r}-\mathrm{g}) 0.08 \&(\mathrm{r}-\mathrm{b})>0.08$; blue: $(\mathrm{b}-\mathrm{g})>0 / 01 \&(\mathrm{~b}-\mathrm{r})$; yellow:9r-b) $>0.12 \&(\mathrm{~g}-\mathrm{b})>0.12$. The pixels that are not in the range above are considered as other colours. The three colours in the range are then segmented and the results converted to binary images. If the colour is within this range, set the pixel area to white, otherwise black. 


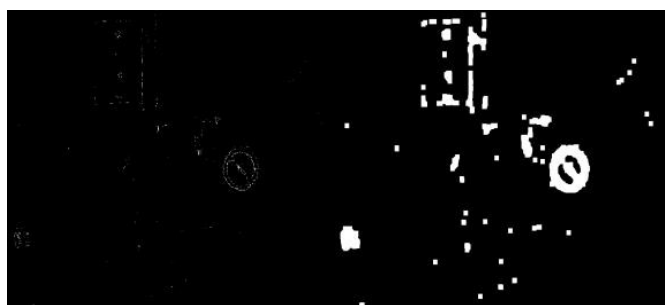

Fig 3. (c)Binary image (d) Image after expansion

\subsection{Expansion of Road Sign Image}

The expansion of image has the best effect on binary image. Moreover, this is the edge operation of the image, which can make the target area larger, and make the target more operational, and is helpful for edge extraction and other operations on the target. Compared with the previous image, it can be found that the road sign and its position become clearer after the expansion of the binary image. Therefore, it can be found that expansion is conducive to the position recognition of road sign. See figure (d).

\subsection{Filling of Road Sign Image}

The filling operation [26] starts from the point specified in the position to perform the flood filling operation on the background pixel of the input binary image BW. If the position is a p-by-1 vector, it contains a linear index of the starting position. If the position is a p-by-ndims (BW) matrix, each row contains an array index of the starting position.

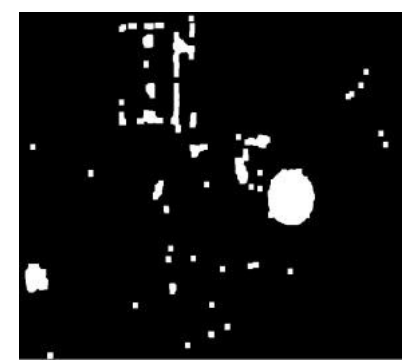

Fig 4. (e) Image after filling

\subsection{The Removal of Non-target Area of Road Signs and Identification of Area of Interest}

The mathematical morphology is used for image processing and pattern recognition. It can measure and extract the corresponding shapes in the images with a certain shape of the organization element graph, so as to achieve the purpose of image analysis and recognition and effectively remove noise. In this way, it conducts image erosion and removes the impurity of the pre-processed images. Then the small non-target area is removed according to the binary traffic sign image. By using the shape feature of the sign to eliminate the non-target position, the relatively accurate position of the traffic sign can be obtained finally.

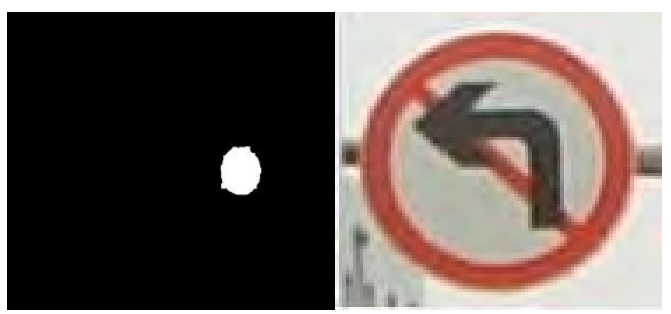

Fig 5. (f) Target position (g) Road sign image 


\section{Traffic Sign Recognition}

\subsection{Basic Principles of LBP Operator}

LBP is an effective operator to describe local texture features of images, which was first proposed by T. Ojala et.al in 1994 for texture feature extraction. It is an operation based on neighbourhood pixels. Its core idea is to binarize the neighbourhood pixels by taking the centre pixel as a threshold, and obtain a binary string to represent the LBP texture features of the centre pixel.

The original LBP operator is calculated by taking the template of $3 * 3$ as the neighbourhood region, that is, taking the central pixel value of the template as the threshold. Compare the pixel value of 8neighbourhood with the centre pixel value, and the surrounding pixel value is less than the gray value of the centre pixel, then the pixel position is marked as 0 ; otherwise, it is marked as 1 . An LBP binary string is obtained, which is converted to decimal number, namely the LBP value of the centre pixel, as shown in the following process:

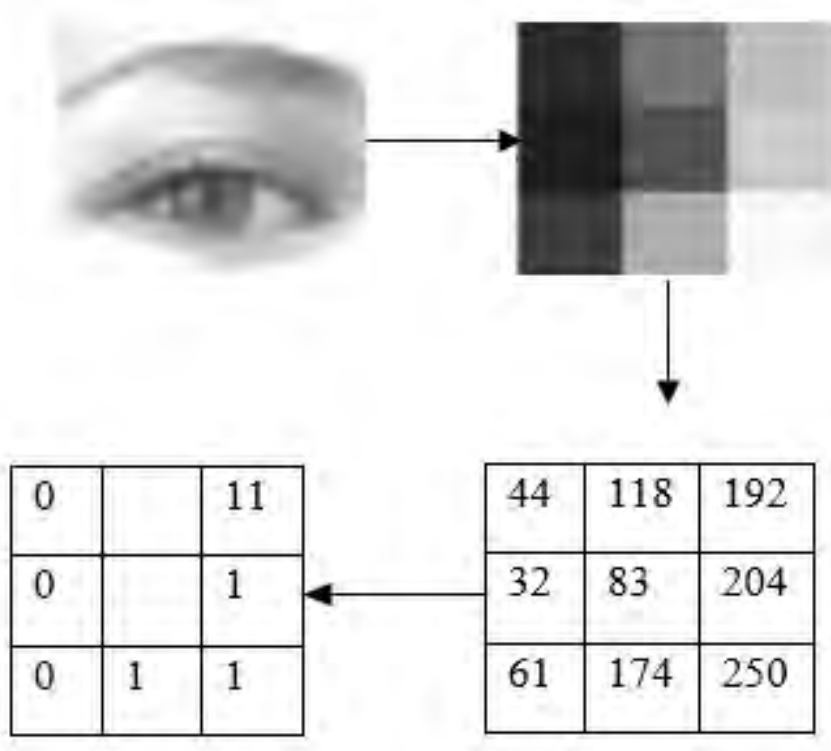

Fig 6. Shown in the following process

Where, the LBP value is: $(01111100)_{10}=124$. By collecting the LBP of each pixel segmentation, a histogram can be established. The type of each LBP is the horizontal axis, and the number of occurrences of each LBP is the vertical axis as shown in the following figure.
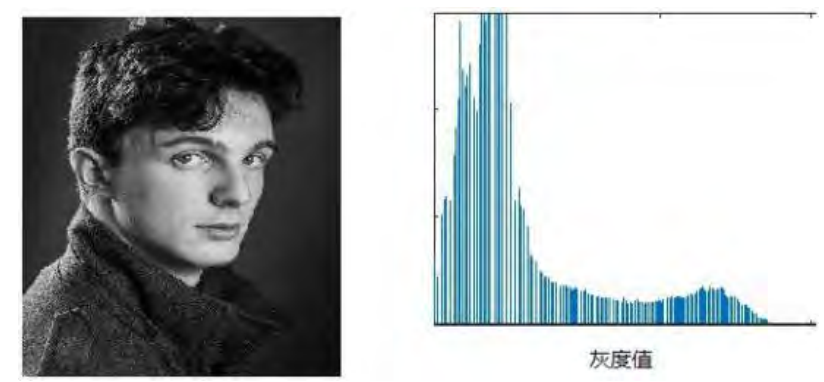

Fig 7. From this histogram, we can get the texture information of this graph.

\subsection{Basic Principles of SVM}

The support vector machine was first proposed by Cortes and Vapnik in 1995. It shows many unique advantages in solving small-sample, non-linear and high dimension pattern recognition, and can be applied to other machine learning problems such as function fitting.

SVM has strong advantages in small-sample, non-linear and high dimension sample classification. In the two types of classification problems that can be classified in advance, we hope to find an 
optimal plane to make the data point interval between the two classes maximum. We call this plane the maximum interval hyperplane. SVM constructs the decision function by looking for such a maximum interval hyperplane, as shown in the figure below:

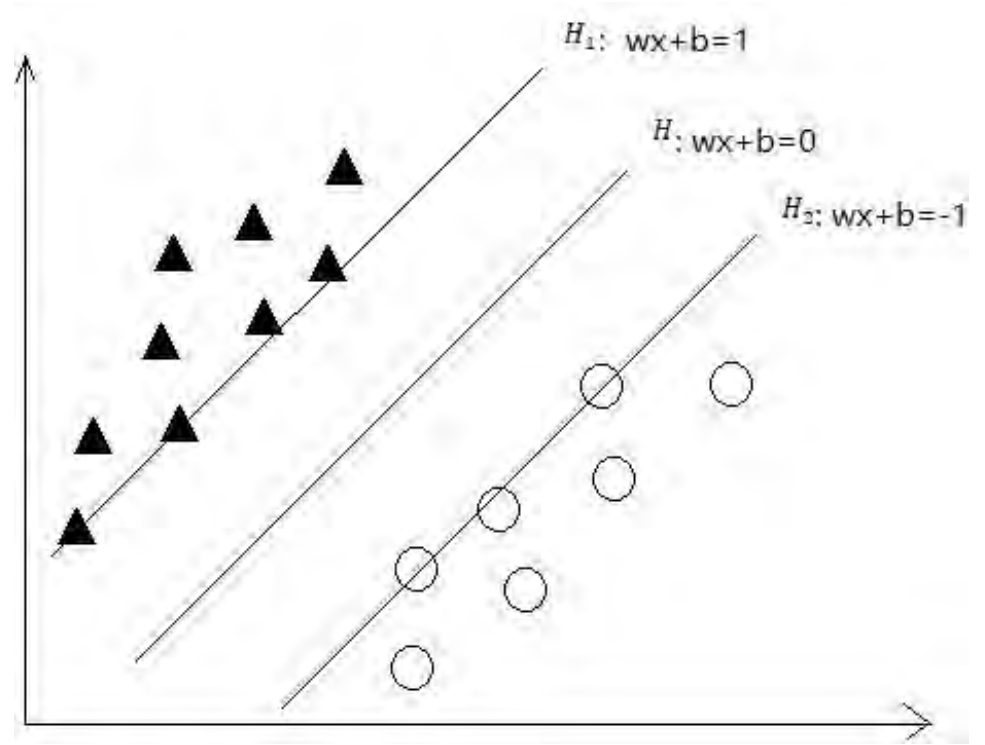

Fig 8. SVM constructs the decision function by looking for such a maximum interval hyperplane

There are now two types of samples: black triangle sample a and white circular sample $b$. We can find a line that completely separates type a from type $b$, and we can find a line in the sample of type $a$ that is the closest. Similarly, you can find a line in type b, and three lines are parallel to each other. The shortest distance between $\mathrm{H} 1$ and $\mathrm{H} 2$ is denoted as Margin, which is called boundary distance. SVM classifier is designed to find a line that can completely separate the two types of samples and make Margin reach the maximum. If you have two types of samples, and $\left(x_{1}, y_{1}\right)$, $\left(x_{2}, y_{2}\right) \ldots\left(x_{l}, y_{l}\right)$ is used to represent, where 1 represents the number of samples, $\mathrm{x}$ is the observation data, and $y \in(-1,+1)$ is sample type label $(-1,+1)$. We need to find an optimal hyperplane in a space to separate the two known samples completely.

This function can be expressed as:

$$
f(x)=\operatorname{sgn}\left\{\left(w^{*} \cdot x\right)+b\right\}=\operatorname{sgn}\left\{\sum_{i=1}^{l} \alpha_{i}^{*} y_{i}\left(x_{i} \cdot x\right)+b^{*}\right\}
$$

Where, $b^{*}$ is the bias constant term, which can be obtained by using any support vector with the formula:

$$
y_{i}\left(w^{*} \cdot x_{i}+b^{*}\right)=1
$$

\section{Experiment Results and Conclusion}

Through the traffic sign recognition algorithm based on SVM of LBP, the traffic sign is finally recognized. The results are as follows:

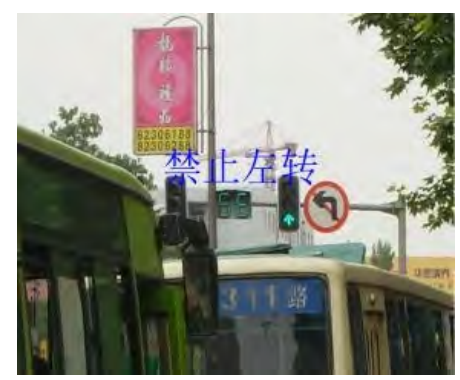

Fig 9. (h) Recognition result 
In this paper, the target image is first transformed from RGB space to HSV space for machine recognition. After image binarization, expansion and filling, the determination of target area has been completed. Finally, LBP operator is used to obtain the texture features of the image, and SVM method is used to train, recognise and classify the features. After verifying the effectiveness of the recognition method in the traffic sign database, it is proved that the recognition algorithm of traffic sign presented in this paper is accurate, effective and feasible.

\section{Acknowledgements}

Thank for the support of the National Natural Science Foundation of China (Item NO. 61773360).

\section{References}

[1]. Yiming Lei, Ximei Zhao, Guodong Wang, Kexin Yu, Weidong Guo. A novel approach for cirrhosis recognition via improved LBP algorithm and dictionary learning. Biomedical Signal Processing and Control, Volume 38, September 2017, Pages 281-292.

[2]. Vivek H. Mahale, Mouad M. H. Ali, Pravin L. Yannawar, Ashok T. Gaikwad, Image Inconsistency Detection Using Local Binary Pattern (LBP)Procedia Computer Science, Volume 115, 2017, Pages 501-508.

[3]. Ravi Kumar Y B; C N Ravi Kumar Local binary pattern: An improved LBP to extract nonuniform LBP patterns with Gabor filter to increase the rate of face similarity.2016 Second International Conference on Cognitive Computing and Information Processing (CCIP).

[4]. Siti Anis Amirah Mohd Faudzi; Norashikin Yahya. Evaluation of LBP-based face recognition techniques. 2014 5th International Conference on Intelligent and Advanced Systems (ICIAS). Year: 2014 Pages: $1-6$.

[5]. Yan Yang; Juan Wang; Yongyi Yang. Exploiting rotation invariance with SVM classifier for microcalcification detection. 2012 9th IEEE International Symposium on Biomedical Imaging (ISBI). Year: 2012 Pages: 590 - 593.

[6]. Zhou Hao; Li Shaohong; Sun Jinping. Unit Model of Binary SVM with DS Output and its Application in Multi-class SVM. 2011 Fourth International Symposium on Computational Intelligence and Design. Year: 2011, Volume: 1 Pages: $101-104$.

[7]. Elia Moscoso Thompson, Silvia Biasotti. Description and retrieval of geometric patterns on surface meshes using an edge-based LBP approach. Pattern Recognition, Volume 82, October 2018, Pages 1-15.

[8]. Jianfeng Ren, Xudong Jiang, Junsong Yuan Learning LBP structure by maximizing the conditional mutual information. Pattern Recognition, Volume 48, Issue 10, October 2015, Pages 3180-3190.

[9]. M Hakeem Selamat; Helmi Md Rais Image face recognition using Hybrid Multiclass SVM (HMSVM). 2015 International Conference on Computer, Control, Informatics and its Applications (IC3INA). Year: 2015Pages: $159-164$.

[10]. Xiaoming Liu; Jinshan Tang Mass Classification in Mammograms Using Selected Geometry and Texture Features, and a New SVM-Based Feature Selection Method. IEEE Systems Journal Year: 2014, Volume: 8, Issue: 3 Pages: 910 - 920.

[11]. Abeg Kumar Jaiswal, Haider Banka. Local pattern transformation-based feature extraction techniques for classification of epileptic EEG signals. Biomedical Signal Processing and Control, Volume 34, April 2017, Pages 81-92. 
[12]. Hsin-Hsiung Huang; Zijing Wang; Wingyan Chung. Efficient parameter selection for SVM: The case of business intelligence categorization. 2017 IEEE International Conference on Intelligence and Security Informatics (ISI). Year: 2017 Pages: 158 - 160.

[13]. Rui Wang, Wei Li, Rui Li, Liang Zhang. Automatic blur type classification via ensemble SVM. Signal Processing: Image Communication, In press, accepted manuscript, Available online 17 August 2018.

[14]. Li-Li Wang, Henry Y. T. Ngan, Nelson H. C. Yung. Automatic incident classification for large-scale traffic data by adaptive boosting SVM. Information Sciences, Volume 467, October 2018, Pages 59-73.

[15]. A. Møgelmose, D. Liu, and M. M. Trivedi, "Detection of U.S. traffic signs," IEEE Trans. Intell. Transp. Syst., vol. 16, no. 6, pp. 3116-3125, Dec. 2015.

[16]. M. Mathias, R. Timofte, R. Benenson, and L. Van Gool, "Traffic sign recognition-How far are we from the solution?" in Proc. Int. Joint Conf. Neural Netw. (IJCNN), Aug. 2013, pp. 1-8.

[17]. Z. Huang, Y. Yu, S. Ye, and H. Liu, "Extreme learning machine-based traffic sign detection," in Proc. Int. Conf. Multisens. Fusion Inf. Integr. Intell. Syst. (MFI), 2014, pp. 1-6.

[18]. G. Wang, G. Ren, Z. Wu, Y. Zhao, and L. Jiang, "A robust, coarseto-fine traffic sign detection method," in Proc. Int. Joint Conf. Neural Netw. (IJCNN), 2013, pp. 1-5.

[19]. Wang fangshi, Wang jian, Li bing, et al. Traffic sign detection based on deep attribute learning [J] journal of jilin university (engineering edition), 2018(1):319-329. 